\title{
Anaesthetic gel to reduce pain during periodontal scaling and root planing
}

\author{
When people undergo periodontal scaling and root planning, is an anaesthetic \\ gel effective in producing pain relief?
}

\section{Donaldson D, Gelskey SC, Landry RG, Matthews DC, Sandhu \\ HS. A placebo-controlled multi-centred evaluation of an anaesthetic gel (Oraqix ${ }^{\mathbb{}}$ ) for periodontal therapy. Clin Periodontol 2003; 30:171-175}

Design The study was a multicentre randomised controlled trial (RCT).

Intervention A total of 130 patients received either the active or placebo gel in periodontal pockets in one quadrant of the mouth for $30 \mathrm{~s}$, prior to periodontal debridement (scaling and root planing).

Outcome measure Pain was measured using both a $100-\mathrm{mm}$ visual analogue scale (VAS) and a verbal rating scale (VRS).

Results The median VAS pain score for people treated with the anaesthetic gel was $5 \mathrm{~mm}$ (range, $0-5 \mathrm{~mm}$ ) as opposed to $13 \mathrm{~mm}$ (range, $0-9 \mathrm{~mm})$ in the placebo-treated patients $(P=0.015)$. There was no significant difference in the proportion of patients who reported no pain or mild pain $(78 \%$ and $76 \%$ for the anaesthetic gel and placebo, respectively).

Conclusions The VAS pain scores showed that the anaesthetic gel $5 \%$ was statistically more effective than the placebo in reducing pain during periodontal debridement.

Acknowledgements The study was supported by Astra Zeneca Pain Control Sweden.

\section{Commentary}

The fear and discomfort so often associated with injection of local anaesthetics in dentistry has generated a desire for "needle-less" pain relief. The solution, topical anaesthetic products, is not new to clinical practice but a number of new gels, often containing a mixture of anaesthetic agents, have emerged recently. This study aimed to evaluate one of these for use in periodontal therapy.

At first sight, the study appears to have fulfilled all the criteria required for a clinical RCT. Information is provided about subject and site selection, randomisation and sample size calculation as well as use of a control group. A number of questions remain to be answered, however, a point apparently not lost on the authors who admit that, "further studies are required to confirm its true efficacy." Perhaps the greatest problem in this sort of study is the subjective nature of pain assessment, combined here with an unquantified placebo effect. In fact, no mention is made of the nature of the placebo and, as many subjects will have already experienced the use of topical anaesthetics, it may have been possible for individuals to identify whether they had received test or control product. In any case, the VAS scores for pain were generally low in both groups; no subjects felt that periodontal debridement produced pain described as "the worst imaginable", such that only a very small number required "rescue" anaesthetics. Indeed, the rather bland statement that, "a large proportion of scaling and debridement procedures involve nerve block or infiltration anaesthesia" is open to question. It is therefore unfortunate that a number of variables that might influence the level of pain experienced were not reported on. Thus, periodontal diagnosis (chronic or aggressive disease), the degree of soft tissue inflammation present and smoking history may well be relevant.

Statistically, one might question the method of sample size calculation, given that six separate centres were used in this trial. This may be an important issue for multicentre trials where data are pooled. Indeed, the authors accept that the type of subject did vary between centres and they attributed the disparity in results obtained to this fact. On a similar subject, the inclusion of three fairly unconvincing scatter diagrams without any reference to statistical significance and described in the text as an "exploratory analysis", leads to a comment on trends with conclusions that are difficult to justify.

The development of a useful anaesthetic gel would be a great help to a significant number of patients. It is unfortunate that this research has not answered the question of whether it is effective in this case. Pain is not an easy topic to investigate and this study appears not to have overcome this difficulty. Without doubt, more research is needed.

\section{Graham Smart}

Specialist on Periodontics, London and Oxford, and Postgraduate Dental Tutor for Buckinghamshire, UK

Evidence-Based Dentistry (2003) 4, 78.

doi:10.1038/sj.ebd.6400218
Address for correspondence: Dr David Donaldson, Department of Oral Biological and Medical Sciences, Faculty of Dentistry, The University of British Columbia, Vancouver, Canada. E-mail: ddonald@interchange.ubc.ca. 